\title{
Application of metamorphic theory in vehicle shift dynamics
}

\begin{abstract}
The present researches of vehicle shift dynamics are mutually independent and essentially qualitative. It is quite difficult to characterize the dynamics behaviors of vehicle transmission shift systematically, integrally and quantitatively. Vehicle power train is one kind of the generalized metamorphic mechanism. Therefore, it is necessary to pursue the study of vehicle shift dynamics to break through the limit of the present research contents and modeling methods based on the metamorphic theory. This research offers the theory and technology support for the study of shift optimization, dynamic strength design and vehicle comfort.
\end{abstract}

Keywords: vehicle gear shift, metamorphic theory, variable topology structure, automatic transmission
Volume 3 Issue I - 2019

\author{
Fu Shengping, Li Shengbo, Luo Ning \\ School of mechanical and vehicle Engineering, Xiamen University \\ of Technology, China
}

\begin{abstract}
Correspondence: Fu Shengping, Xiamen University of Technology, No. 600, Ligong Rd., Jimei District, Xiamen, Fujian, PR, China, Tel 86-18850339235, Fax (86 592)629|386, Email7025370@।63.com
\end{abstract}

Received: January 25, 2018 | Published: February 21, 2019

\section{Introduction}

The present researches of vehicle shift dynamics are mutually independent and essentially qualitative. ${ }^{1,2}$ It is quite difficult to characterize the dynamics behaviors of vehicle transmission shift systematically, integrally and quantitatively. Therefore, it is necessary to pursue the study of vehicle shift dynamics to break through the limit of the present research contents and modeling methods based on the metamorphic theory.

\section{Method}

Metamorphic theory interpret how the topological structure and mechanism freedom change with the boundary conditions variation. The essence of the vehicle shift is the transformation from one kind of stable state to another according to the variation of the transmission parts, constraints, and power transmission route etc. This process behaves the characteristics of multi-function stage transformation, multi-freedom variation, multi topological mechanism. Therefore, vehicle shift process conforms to the metamorphic mechanism theory. The study of vehicle shift dynamics based on the metamorphic theory can be decomposed as following. Firstly, the power train is gradually disassembled to hierarchical models. Secondly, metamorphic route and metamorphic form of each vehicle shift are mathematically presented. And the nonlinear mechanism models of gear pair and wet clutch are both established. Thirdly, the metamorphic function and metamorphic matrix of vehicle shift are established based on the set theory and screw theory.

\section{Discussion}

Most present researches on the topology analysis of the vehicle power train are summarized as following. References, ${ }^{3,4}$ analyzed and synthesized the topology structure of gear transmission system based on graph theory. These studies only took the topology structure of each component in the gearbox into consideration. Topology description was irrelative with motion pair types and poses. It resulted that one kind of graph theory model demonstrated topology structures of several different types of gearbox. And the graphical description of shifting process was absolutely blank based on graph theory. References,${ }^{5,6}$ deeply studied topology structures of fixed-axle and planetary gear train in dynamics adopting bond graph theory. However, spatial characteristics of gearbox each component and joint were not adequately interpreted in these studies. It was not significant and fully targeted for mathematics mapping and graph mechanisms of spatial topology structure adopting empirical method, classical mathematic and mechanics. ${ }^{7,8}$ And these methods were not practical. Therefore, to solve problems of absent systematic topology model and fuzzy topology definition of vehicle power train, it is necessary to non-numerically definite and describe variable topology structure of vehicle power train based on metamorphic theory. And shifting process is directly described by graphs.

\section{Conclusion}

Vehicle power train is one kind of the generalized metamorphic mechanism. Vehicle shift dynamics is equivalent to the problems of the topological representation, metamorphic principle analysis, and metamorphic equation establishment and solution. This research offers the theory and technology support for the study of shift optimization, dynamic strength design and vehicle comfort.

\section{Acknowledgments}

Supported by a project grant from National Natural Science Foundation of China (Grand No. 51505402).

\section{Conflicts of interest}

The authors declare there is no conflict of interests.

\section{References}

1. Wouter Ost, Patrick De Baets, Mia Loccufier, et al. Torsional vibrations of wet clutches: influence of mechanical parameters. Meccanica. 2010;45(3):341-353.

2. Liu Hui, Xiang Changle, Sun Tiantian. Construction of bending-torsional coupled vibration model and complex modal analysis of the vehicle powertrain. Journal of Mechanical Engineering. 2010;46(24):67-74.

3. Yang Yalian, Mi Jiao, Hu Xiaosong, et al. Graph theory modeling and dynamics analysis on the coupled planetary transmission system of HEV. Automotive Engineering. 2015;37(01):9-15.

4. Xue Huiling, Liu Geng, Yang Xiaohui. Kinetic analysis of planetary gear transmission. Machinery \& Electronics. 2016;34(9):3-6.

5. Wang Zhongshuang, Zhao Yanwei, Tao Yangyang, et al. Vector bond graph method of kineto-static analysis for planar multi-body system with gear pair. Journal of Mechanical Transmission. 2015;39(6):35-38. 
6. Dou ZC, Li YN, Du MG, et al. Dynamics modeling and power flow analysis of the planetary gear based on the bond graph. Chongqing University. Proceedings of the International Conference on Power Transmissions. Chongqing, P.R. China: Power Engineering; 2016. p. 623-630
7. Zou Huijun, Gao Feng. Advances in modern mechanism, 2nd edn. Beijing: China Higher Education Press; 2011.

8. Zeng DX $\square$ Huang Z. Type synthesis of the rotational decoupled parallel mechanism based on screw theory. Science China(Technological Sciences). 2011;54(4):998-1004. 